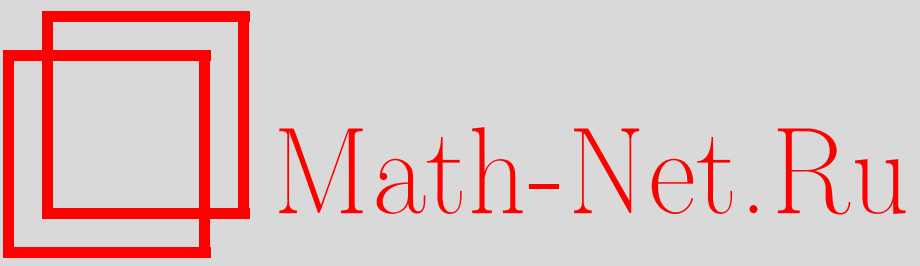

А. В. Докучаев, А. П. Котенко, Алгоритмы решения стохастических задач динамического программирования большой размерности, Вестн. Сам. гос. техн. ун-та. Сер. Физ.-мат. науки, 2008, выпуск 2(), 203-209

DOI: https://doi.org/10.14498/vsgtu627

Использование Общероссийского математического портала Math-Net.Ru подразумевает, что вы прочитали и согласны с пользовательским соглашением

http://www.mathnet.ru/rus/agreement

Параметры загрузки:

IP: 54.84 .234 .179

26 апреля 2023 г., 14:13:14 
УДК 519.856, 519.857

\section{АЛГОРИТМЫ РЕШЕНИЯ СТОХАСТИЧЕСКИХ ЗАДАЧ ДИНАМИЧЕСКОГО ПРОГРАММИРОВАНИЯ БОЛЬШОЙ РАЗМЕРНОСТИ}

\section{А.В. Докучаев, А.П. Котенко}

Самарский государственный технический университет, 443100, Самара, ул. Молодогвардейская, 244.

E-mails: docuhaev@mail.ru, ako1959@mail.ru

Предлагаются алгоритмы решения стохастических задач динамического программирования больших размерностей. Рассматривается задача распределения ресурсов.

Ключевые слова: стохастическое динамическое программирование, метод Р. Беллмана, задача распределения ресурсов.

Введение. При моделировании физических (обработка сигналов), химических (моделирование химических реакций), экономических (календарное планирование, распределение инвестиций), биологических (идентификация генов) процессов, в сельском хозяйстве (планирование севооборота), в экологии (решение задачи сохранения запасов леса), а также при решении инженерных задач часто приходится решать различные задачи динамического программирования.

Оптимизационные задачи в стохастической постановке предполагают учёт случайного характера процессов, протекающих в моделируемых системах. Применение того или иного метода оптимизации для решения задачи в стохастической постановке обусловлено спецификой прикладной задачи. В данной работе речь пойдет о принципиальной возможности учёта случайного характера моделируемых процессов в рамках динамического программирования в дискретном времени и о конкретном классе задач, весьма перспективном для применения моделей динамического программирования в стохастической постановке - распределении ресурсов.

1. Общая постановка задачи распределения ресурса. Общую задачу распределения ресурса в качестве задачи динамического программирования в дискретном времени в детерминированной постановке можно представить следующим образом: распределяется ресурс $x=x_{1}+x_{2}+\cdots+x_{i}+\cdots+x_{n}$ $(i=1,2, \ldots, n, n \in \mathbb{N}), 0 \leqslant x_{i} \leqslant x, x \in \mathbb{R}^{+}$по монотонным функциям освоения (откликам) $f_{i}: \mathbb{R}^{+} \rightarrow \mathbb{R}^{+}$с заданным шагом дискретизации распределяемого ресурса $h=x / m\left(m=1,2, \ldots, n ; h \in \mathbb{R}^{+}\right)$с целью максимизации (минимизации) суммарного эффекта $Z=f\left(x_{1}, x_{2}, \ldots, x_{n}\right)=\sum f_{i}\left(x_{i}\right) \rightarrow \operatorname{extr}($ через extr обозначена функция экстремума). Далее будем рассматривать задачу на максимум. Обозначим через $f_{i i}=f_{i}\left(x_{i}\right)$ (левая часть табл. 1 ). Значение $x=0-$ обозначено в табл. 1 за $x_{0}$.

Докучаев Александр Владимирович - аспирант кафедры прикладной математики и информатики Самарского государственного технического университета.

Котенко Андрей Петрович-дочент кафедры прикладной математики и информатики Самарского государственного технического университета; к.ф.-м.н, дочент. 
Представление задачи распределения ресурса

\begin{tabular}{c|c|c|c|c|c|c}
\hline Порция & \multicolumn{5}{|c}{ Функции освоения } \\
\cline { 2 - 7 } ресурса & $f_{1}$ & $f_{2}$ & $\ldots$ & $f_{i}$ & $\ldots$ & $f_{n}$ \\
\hline$x_{0}$ & $f_{01}$ & $f_{02}$ & $\ldots$ & $f_{0 i}$ & $\ldots$ & $f_{0 n}$ \\
$x_{1}$ & $f_{11}$ & $f_{12}$ & $\ldots$ & $f_{1 i}$ & $\ldots$ & $f_{1 n}$ \\
$x_{2}$ & $f_{21}$ & $f_{22}$ & $\ldots$ & $f_{2 i}$ & $\ldots$ & $f_{2 n}$ \\
$\ldots$ & $\ldots$ & $\ldots$ & $\ldots$ & $\ldots$ & $\ldots$ & $\ldots$ \\
$x_{i}$ & $f_{i 1}$ & $f_{i 2}$ & $\ldots$ & $f_{i i}$ & $\ldots$ & $f_{i n}$ \\
$\ldots$ & $\ldots$ & $\ldots$ & $\ldots$ & $\ldots$ & $\ldots$ & $\ldots$ \\
$x_{n}$ & $f_{n 1}$ & $f_{n 2}$ & $\ldots$ & $f_{n i}$ & $\ldots$ & $f_{n n}$ \\
\hline
\end{tabular}

\begin{tabular}{c|c|c|c|c|c|c}
\hline Порция & \multicolumn{6}{|c}{ Функии освоения } \\
\cline { 2 - 7 } ресурса & $f_{1}$ & $f_{2}$ & $\ldots$ & $f_{i}$ & $\ldots$ & $f_{n}$ \\
\hline$y_{0}$ & $f_{01}$ & $f_{02}$ & $\ldots$ & $f_{0 i}$ & $\ldots$ & $f_{0 n}$ \\
$y_{1}$ & $f_{11}$ & $f_{12}$ & $\ldots$ & $f_{1 i}$ & $\ldots$ & $f_{1 n}$ \\
$y_{2}$ & $f_{21}$ & $f_{22}$ & $\ldots$ & $f_{2 i}$ & $\ldots$ & $f_{2 n}$ \\
$\ldots$ & $\ldots$ & $\ldots$ & $\ldots$ & $\ldots$ & $\ldots$ & $\ldots$ \\
$y_{j}$ & $f_{j 1}$ & $f_{j 2}$ & $\ldots$ & $f_{j i}$ & $\ldots$ & $f_{j n}$ \\
$\ldots$ & $\ldots$ & $\ldots$ & $\ldots$ & $\ldots$ & $\ldots$ & $\ldots$ \\
$y_{n}$ & $f_{n 1}$ & $f_{n i}$ & $\ldots$ & $f_{n i}$ & $\ldots$ & $f_{n n}$ \\
\hline
\end{tabular}

Суммарный эффект $Z$, а также взвешенный вектор распределения ресурсов $\left(x_{1}^{*}, x_{2}^{*}, \ldots, x_{n}^{*}\right)$, где $x_{i}^{*}$ - ресурс, при распределении которого функция $f_{i}\left(x_{i}\right)$ обеспечивает оптимум, находятся методом динамического программирования на основе рекуррентных соотношений Р. Беллмана [1] с помощью программного комплекса, созданного авторами данной статьи.

Уточним математическую модель задачи: с учётом распределения $x$ порциями обозначим $k_{i}=x_{i} / h, k_{i}=1,2, \ldots, m$, тогда

$$
g\left(k_{1}, k_{2}, \ldots, k_{n}\right)=\sum f_{i}\left(k_{i} h\right)=\sum f_{i}\left(x_{i}\right)
$$

Если $Z_{\max }=f\left(x_{1}^{*}, x_{2}^{*}, \ldots, x_{n}^{*}\right)$, то $Z_{\max }=g\left(k_{1}^{*}, k_{2}^{*}, \ldots, k_{n}^{*}\right)$, где $k_{i}^{*}=x_{i}^{*} / h$. Обозначим $y_{j}=j h, j=0,1,2, \ldots, m, f_{j i}=f_{i}\left(y_{j}\right)$ (см. правую часть табл. 1 ).

2. Постановка стохастической задачи динамического программирования. Рассмотрим теперь одну стохастическую модель приведенной выше задачи. Пусть некоторые из $f_{i}\left(y_{j}\right)$ являются дискретными случайными величинами с заранее известными носителями $\left[a_{i j} ; b_{i j}\right]\left(a_{i j}, b_{i j} \in \mathbb{R}^{+}\right)$с заданными рядами распределения. В качестве критерия оптимизации используется математическое ожидание суммарного эффекта: $M[Z] \rightarrow \max$.

Для решения стохастической задачи распределения ресурсов на основе метода динамического программирования используется байесовский подход $[2,3]$, при котором стохастическая задача разбивается на конечное множество детерминированных подзадач.

Пусть значение одной из функций освоения $f_{i}\left(y_{j}\right)$ является дискретной случайной величиной с известным рядом распределения. Тогда значениям функции освоения $f_{i t}\left(y_{j}\right)$ соответствуют априорные вероятности $P_{t}\left(f_{i t}\left(y_{j}\right)\right)$, где $t$-количество спектральных точек $(t=1,2, \ldots, m, m \in \mathbb{N})$ данной $f_{i}$ и данной задачи (потому что только одно значение одной рассматриваемой функции представляет собой стохастическую величину). Тогда значение целевой функции будет дискретной случайной величиной, имеющей $t$ спектральных точек. Найдём её математическое ожидание

$$
M[Z]=\sum_{j=1}^{m} Z_{t} P_{t}\left(f_{i t}\left(y_{j}\right)\right),
$$

где $Z_{t}$ - оптимальный суммарный эффект в точке спектра $t$. Если случайны два и более значения одной из функций освоения и (или) значения нескольких функций освоения, то для определения математического ожидания суммарного эффекта используется формула Байеса. 
Таким образом, решая задачу стохастического динамического программирования, мы переходим к процедуре решения конечного множества детерминированных подзадач динамического программирования [2].

При увеличении стохастических параметров задачи вычислительная сложность алгоритма резко возрастает. Непосредственный подсчет математического ожидания оптимального значения представляет значительную трудность и зачастую уже для задач небольших размерностей не является практически реализуемым даже с использованием современных ЭВМ. Поэтому актуальна возможность упрощения такой процедуры.

Существует несколько возможных путей устранения данной проблемы:

1) совершенствование существующих и разработка новых алгоритмов динамического программирования;

2) понижение размерности текущей задачи с помощью методов декомпозиции;

3) применение методов приближенного решения.

Применение процедуры динамического программирования для нахождения оптимального распределения ресурса $X$ приводит к громоздким выкладкам при росте числа уровней дискретизации $k$. На основе континуальной постановки задачи динамического программирования [2] предложим упрощенный метод решения задачи.

3. Упрощенный метод решения стохастической задачи динамического программирования. Решая стохастическую задачу распределения ресурсов методом динамического программирования, в итоге переходим к решению детерминированных подзадач (см. п. 2 настоящей работы).

Для детерминированной подзадачи $\left(x_{1}^{*}, x_{2}^{*}, \ldots, x_{n}^{*}\right)$ - приближённое решение, если для каждой функции $f_{i}\left(y_{j}\right)$ (см. табл. 1) выполняются условия $\Delta f_{1}\left(y_{1}\right) \approx \Delta f_{2}\left(y_{2}\right) \approx \cdots \approx \Delta f_{n}\left(y_{n}\right)$, где $\Delta f_{i}\left(y_{j}\right)=f_{i}\left(y_{j}\right)-f_{i}\left(y_{j-1}\right), \sum x_{i}^{*}=x$.

Если в частном случае $\Delta f_{1}\left(y_{1}\right)=\Delta f_{2}\left(y_{2}\right)=\cdots=\Delta f_{n}\left(y_{n}\right)$, где $\Delta f_{i}\left(y_{j}\right) \neq 0$, то значения $\left(y_{1}, y_{2}, \ldots, y_{n}\right)$ представляют точное решение (идентично находимому динамическим программированием, пример приведен в статье [2]).

В ходе численных экспериментов показано, что если увеличение $h$ не влияет на найденное ранее решение, то есть $f_{i}\left(y_{j}\right): Z\left(f\left(y_{j+1}\right)\right)=Z\left(f\left(y_{j}\right)\right)$ при $m_{j+1}>m_{j}$, то $\lim _{j \rightarrow 0} f\left(y_{j}\right)=\lim _{i \rightarrow+\infty} f\left(x_{i}^{*}\right)$.

Предложенная методика сокращения вычислительного объёма задачи динамического программирования применима как в детерминированной, так и в стохастической постановках задачи распределения ресурсов, когда исходные данные математической модели не известны точно либо являются случайными величинами. Достоверность предложенного метода проверена в ходе серии численных экспериментов.

4. Метод декомпозиции. При решении задач динамического программирования зачастую возникают непреодолимые вычислительные сложности (в рассматриваемой задаче объём вычислений растёт при уменьшении шага дискретизации, при увеличении количества функций освоения, при возрастании числа случайных параметров), поэтому актуален вопрос о понижении размерности задачи. Получена декомпозиция метода динамического программирования по монотонным функциям освоения $f(x): \mathbb{R}^{+} \rightarrow \mathbb{R}^{+}$в задаче распределения ресурса $x$. На основе рассматриваемой постановки задачи предлагается классификация случаев, предполагающих различные алгоритмы деком- 
позиции в зависимости от вида и количества оптимальных траекторий $x^{*}$ : $\sum_{i=1}^{n} x_{i}^{*}=x$. Основная идея предлагаемого метода заключается в группировании похожих по поведению в точках дискретизации функций освоения.

Рассмотрим пример. Пусть для определенности $i=2 n$. Предложим алгоритм декомпозиции для задачи с данными, которые приведены в табл. 2.

$$
\text { Таблица } 2
$$

Исходные данные задачи

\begin{tabular}{c|c|c|c|c|c|c}
\hline$x$ & $f_{1}(x)$ & $f_{2}(x)$ & $f_{3}(x)$ & $f_{4}(x)$ & $f_{5}(x)$ & $f_{6}(x)$ \\
\hline 0 & 0 & 0 & 0 & 0 & 0 & 0 \\
1 & 34 & 30 & 32 & 31 & 30 & 30 \\
2 & 64 & 58 & 63 & 61 & 57 & 55 \\
3 & 90 & 84 & 93 & 89 & 82 & 77 \\
4 & 112 & 108 & 122 & 115 & 106 & 96 \\
5 & 130 & 130 & 150 & 139 & 128 & 112 \\
6 & 146 & 150 & 177 & 161 & 149 & 125 \\
7 & 158 & 168 & 203 & 181 & 168 & 135 \\
8 & 166 & 184 & 228 & 199 & 186 & 142 \\
9 & 172 & 198 & 252 & 215 & 202 & 146 \\
10 & 174 & 210 & 275 & 229 & 217 & 117 \\
\hline
\end{tabular}

а. Понизим размерность задачи в два раза. Для этого произведём равномерную группировку $f(x)$, то есть суммируем значения функций освоения $f_{1}(x)$ с $f_{2}(x), f_{3}(x)$ с $f_{4}(x)$ и $f_{5}(x)$ с $f_{6}(x)$ (считается, что функции попарно похожи, в более общем случае группировка функций неравномерна).

b. Облегчённая задача решается стандартным методом динамического программирования: получаем ресурсы, распределяемые на функции $f_{i / 2}^{l}(x)=$ $=f_{i-1}^{l}(x)+f_{i}^{l}(x), l=2,3, \ldots$ (показывает, во сколько раз понижена размерность исходной задачи).

с. Перейдём к решению $i / 2=n$ подзадач обычным методом динамического программирования.

Выделен и исследован частный случай при условиях:

1) для всех решаемых подзадач $x_{i}^{*} \neq 0$ - единственное решение;

2) в решении исходной задачи отсутствуют альтернативные траектории (под траекторией понимается взвешенный вектор распределения ресурсов, соответствующий оптимуму);

3) в решении исходной задачи отсутствуют нулевые траектории.

УтвеРжДЕние 1. Оптималъные траектории подзадач - точное решение исходной задачи. Z находится склейкой решений подзадач.

Суммарный эффект, получаемый методом динамического программирования для детерминированной задачи распределения ресурса, соответствующей данным табл. 3, составил 308 единиц, взвешенный вектор распределения ресурсов - $(2,1,3,2,1,1)$. При группировании функций $f_{1}$ с $f_{2}, f_{3}$ с $f_{4}$ и $f_{5}$ с $f_{6}$ получаем 3 подзадачи.

Из столбцов $\left(f_{1}(x)+f_{2}(x)\right),\left(f_{3}(x)+f_{4}(x)\right)$ и $\left(f_{5}(x)+f_{6}(x)\right)$ составляем новую таблицу данных задачи и решаем преобразованную задачу методом динамического программирования. В результате получаем, что на функции 
Таблица 3

Разбиение исходной задачи на 3 подзадачи

\begin{tabular}{c||c|c|c||c|c|c||c|c|c}
\hline$x$ & $f_{1}(x)$ & $f_{2}(x)$ & $f_{1}+f_{2}$ & $f_{3}(x)$ & $f_{4}(x)$ & $f_{3}+f_{4}$ & $f_{5}(x)$ & $f_{6}(x)$ & $f_{5}+f_{6}$ \\
\hline 0 & 0 & 0 & 0 & 0 & 0 & 0 & 0 & 0 & 0 \\
1 & 34 & 30 & 64 & 32 & 31 & 63 & 30 & 30 & 60 \\
2 & 64 & 58 & 122 & 63 & 61 & 124 & 57 & 55 & 112 \\
3 & 90 & 84 & 174 & 93 & 89 & 182 & 82 & 77 & 159 \\
4 & 112 & 108 & 220 & 122 & 115 & 237 & 106 & 96 & 202 \\
5 & 130 & 130 & 260 & 150 & 139 & 289 & 128 & 112 & 240 \\
6 & 146 & 150 & 296 & 177 & 161 & 338 & 149 & 125 & 274 \\
7 & 158 & 168 & 326 & 203 & 181 & 384 & 168 & 135 & 303 \\
8 & 166 & 184 & 350 & 228 & 199 & 427 & 186 & 142 & 328 \\
9 & 172 & 198 & 370 & 252 & 215 & 467 & 202 & 146 & 348 \\
10 & 174 & 210 & 384 & 275 & 229 & 504 & 217 & 147 & 364 \\
\hline
\end{tabular}

освоения $\left(f_{1}(x)+f_{2}(x)\right)$ распределяется 3 единицы ресурса $x$, на $\left(f_{3}(x)+\right.$ $\left.+f_{4}(x)\right)-5$ единиц и на $\left(f_{5}(x)+f_{6}(x)\right)-2$ единицы. Теперь переходим к решению получившихся подзадач.

Решая каждую из подзадач методом динамического программирования, получаем решения: $(2,1),(3,2),(1,1)$. Склеиваем найденные решения и сравниваем с полученным при применении стандартного метода динамического программирования. Они оказываются идентичными.

Пусть теперь соблюдаются только условия 1), 2).

Утверждение 2. Если решение одной из подзадач $x_{i}^{*} \in \min \left\{f_{1}(x), f_{2}(x), \ldots\right.$, $\left.f_{i}(x), \ldots, f_{n}(x)\right\}$, то решение исходной задачи будет приближённым; данная точка будет нулевой компонентой взвешенного вектора распределения ресурса исходной задачи (при нахождении методом динамического программирования).

В качестве примера рассмотрим задачу с исходными данными в табл. 4.

Для данной задачи найден взвешенный вектор распределения ресурсов $(2,1,1,1,3,2)$, ему соответствует суммарный эффект 311 единиц. Решение

Таблица 4

Исходные данные задачи

\begin{tabular}{c|c|c|c|c|c|c}
\hline$x$ & $f_{1}(x)$ & $f_{2}(x)$ & $f_{3}(x)$ & $f_{4}(x)$ & $f_{5}(x)$ & $f_{6}(x)$ \\
\hline 0 & 0 & 0 & 0 & 0 & 0 & 0 \\
1 & 29 & $\mathbf{2 8}$ & 29 & 29 & 36 & 34 \\
2 & 57 & 55 & 56 & 54 & 70 & 66 \\
3 & 84 & 80 & 81 & 76 & 102 & 96 \\
4 & 110 & 103 & 104 & 95 & 132 & 123 \\
5 & 135 & 124 & 125 & 111 & 160 & 147 \\
6 & 159 & 143 & 144 & 124 & 186 & 168 \\
7 & 182 & 160 & 161 & 134 & 210 & 186 \\
8 & 204 & 175 & 176 & 141 & 232 & 201 \\
9 & 225 & 188 & 189 & 145 & 252 & 213 \\
10 & 245 & 199 & 200 & 146 & 270 & 222 \\
\hline
\end{tabular}


было найдено по предложенному методу декомпозиции - исходная задача разбивалась аналогично предыдущему примеру с данными в табл. 2. Вторая компонента взвешенного вектора распределения ресурсов попала на локальный минимум по строке в таблице данных исходной задачи (в табл. 4 соответствующее $f_{2}(x)$ выделено жирным шрифтом). Значит, в соответствии с высказанным утверждением 2 полученное решение будет приближённым, а при нахождении методом динамического программирования значение $f_{2}(1)$ можно не учитывать. При нахождении решения динамическим программированием получаем вектор $(1,0,1,1,4,3)$, суммарный эффект 315 единиц. Утверждения 1) и 2) подтверждаются многочисленными примерами, рассмотренными авторами данной работы.

В нерассмотренных случаях решение будет приближённым, их целесообразнее рассматривать отдельно. В результате численных экспериментов установлено, что при неубывающих $f_{i}$, как правило, $Z<Z^{*}$, то есть процедура оптимизации в таком случае приводит нас к локально-оптимальному решению.

5. Исключение функций освоения, не находящихся на критическом пути. При увеличении стохастических параметров задачи вычислительная сложность алгоритма резко возрастает: когда в рассматриваемой постановке задачи все или большая часть $f_{i}\left(y_{j}\right)$ (см. табл. 1) являются случайными, разбивая стохастическую задачу на множество детерминированных подзадач, приходится решать очень много получившихся подзадач. Для сокращения объёма вычислений было предложено [4] перед разбиением исходной задачи произвести усреднение исходных данных с целью последующей оценки влияния исходных данных на конечный результат.

Анализ результатов. Задачи распределения ресурсов представляют собой целый класс задач динамического программирования. Предложенные алгоритмы могут использоваться для решения детерминированных и стохастических (в сочетании с байесовским подходом) задач распределения ресурсов больших размерностей.

Исследовано влияние параметров задачи на математическое ожидание суммарного эффекта в стохастической задаче динамического программирования. В ходе численных экспериментов подтверждены полученные ранее результаты [2-4].

Все полученные результаты необходимо учитывать при построении и адаптации алгоритмов решения стохастических задач распределения ресурсов для минимизации вычислительных трудностей. При решении прикладных задач целесообразно комбинировать предложенные алгоритмы и методы сокращения вычислительных сложностей.

\section{БИБЛИОГРАФИЧЕСКИЙ СПИСОК}

1. Беллман, Р. Динамическое программирование [Текст] / Р. Беллман. - М.: Изд-во ин. лит., 1960. - 400 с.

2. Докучаев, A.B. Адаптивный подход в одной стохастической задаче динамического программирования на основе континуальной постановки [Текст] / А. В. Докучаев / Мат. моделирование и краевые задачи: Тр. пятой всерос. научн. конф. с международ. участием. - Самара: СамГТУ, 2008. - Ч. 2: Моделирование и оптимизация динамических систем и систем с распределёнными параметрами. - С. 25-30. - ISBN 978-5-7964-1088-2.

3. Докучаев, А.В. Использование байесовского подхода в стохастической постановке задачи динамического программирования для оценки влияния погрешностей исходных 
данных [Текст] / А. В. Докучаев / XXXIV Гагаринские чтения: Тр. Международн. молодёжн. научн. конф. - М., 2008. - С. 54-55.

4. Докучаев, A. В. Решение задачи календарного планирования производства в условиях стохастической неопределенности параметров [Текст] / А. В. Докучаев, А. П. Котенко // Вестн. Сам. гос. техн. ун-та. Сер. Физ.-мат. науки. - 2007. - № 2 (15). - С. 182-183.

Поступила в редакцию 30/IX/2008;

в окончательном варианте $-07 / \mathrm{X} / 2008$.

MSC: 90C39, 90C15

\section{NEW ALGORITHM OF DYNAMIC PROGRAMMING FOR STOCHASTIC PROBLEMS SOLUTION}

\section{A. V. Dokuchaev, A.P. Kotenko}

Samara State Technical University,

443100, Samara, Molodogvardeyskaya str., 244.

E-mails: docuhaev@mail.ru, ako1959@mail.ru

Article proposes algorithms for solution of stochastic problems of dynamic programming of the big dimensions. The problem of distribution of resources is studied.

Key words: stochastic dynamic programming, method of R.Bellman, resources distribution problem.

Original article submitted 30/IX/2008; revision submitted $07 / \mathrm{X} / 2008$.

Dokuchaev Alexander Vladimirovich, Post graduate student, Dept. of Applied Mathematics and Computer Science of Samara State Technical University.

Kotenko Andrey Petrovich, Ph.D. (Phis. $E$ S Math.) Assist. Prof., Dept. of Applied Mathematics and Computer Science of Samara State Technical University. 